\title{
A Novel Feistel Cipher Involving a Bunch of Keys supplemented with Modular Arithmetic Addition
}

\author{
Dr. V.U.K Sastry \\ Dean R\&D, Department of Computer Science and \\ Engineering, Sreenidhi Institute of Science \& Tech. \\ Hyderabad, India
}

\begin{abstract}
In the present investigation, we developed a novel Feistel cipher by dividing the plaintext into a pair of matrices. In the process of encryption, we have used a bunch of keys and modular arithmetic addition. The avalanche effect shows that the cipher is a strong one. The cryptanalysis carried out on this cipher indicates that this cipher cannot be broken by any cryptanalytic attack and it can be used for secured transmission of information.
\end{abstract}

Keywords- encryption; decryption; cryptanalysis; avalanche effect; modular arithmetic addition.

\section{INTRODUCTION}

In the development of block ciphers in cryptography, the study of Feistel cipher and its modifications is a fascinating area of research. In a recent investigation [1], we have developed a novel block cipher by using a bunch of keys, represented in the form of a matrix, wherein each key is having a modular arithmetic inverse. In this analysis, we have seen that the multiplication of different keys with different elements of the plaintext, supplemented with the iteration process, has resulted in a strong block cipher, this fact is seen very clearly by the avalanche effect and the cryptanalysis carried out in this investigation.

In this paper, we have modified the block cipher developed in [1] by replacing the XOR operation with modular arithmetic addition. Here our interest is to study how the modular arithmetic addition influences the iteration process and the permutation process involving in the analysis.

In what follows, we present the plan of the paper. In section 2 , we deal with the development of the cipher and introduce the flow charts and the algorithms required in this analysis. We have illustrated the cipher in section 3, and depicted the avalanche effect. Then in section 4, we carry out the cryptanalysis which establishes the strength of the cipher. Finally, we have computed the entire plaintext by using the cipher and have drawn conclusions obtained in this analysis.

Development Of The Cipher

Consider a plaintext containing $2 \mathrm{~m} 2$ characters. Let us represent this plaintext in the form of a matrix $\mathrm{P}$ by using

\author{
Mr. K. Anup Kumar \\ Associate Professor, Department of Computer Science \\ and Engineering, Sreenidhi Institute of Science \& Tech. \\ Hyderabad, India
}

EBCIDIC code. We divide this matrix into two square matrices $\mathrm{P} 0$ and $\mathrm{Q} 0$, where each one is matrix of size $\mathrm{m}$.

The equations governing this block cipher can be written in the form

$\left[P_{j k}^{i}\right]=\left[e_{j k} Q_{j k}^{i-1}\right] \bmod 256$,

$\left[\mathrm{Q}_{\mathrm{jk}}{ }^{\mathrm{i}}\right]=\left(\left[\mathrm{e}_{\mathrm{jk}} \mathrm{P}_{\mathrm{jk}}{ }^{\mathrm{i}-1}\right] \bmod 256+\left[\mathrm{Q}_{\mathrm{jk}}{ }^{\mathrm{i}-1}\right]\right) \bmod 256$,

where $\mathrm{j}=1$ to $\mathrm{m}, \mathrm{k}=1$ to $\mathrm{m}$ and $\mathrm{i}=1$ to $\mathrm{n}$, in which $\mathrm{n}$ is the number of rounds.

the equations describing the decryption are obtained in the form

$\left.\underset{\text { and }}{\left[\mathrm{Q}_{\mathrm{jk}}^{\mathrm{i}-1}\right.}\right]=\left[\mathrm{d}_{\mathrm{jk}} \mathrm{P}_{\mathrm{jk}}^{\mathrm{i}}\right] \bmod 256$,

$\left[\mathrm{P}_{\mathrm{jk}}^{\mathrm{i}-1}\right]=\left[\mathrm{d}_{\mathrm{jk}}\left(\left[\mathrm{Q}_{\mathrm{jk}}{ }^{\mathrm{i}}\right]-\left[\mathrm{Q}_{\mathrm{jk}}^{\mathrm{i}-1}\right]\right)\right] \bmod 256$

where $\mathrm{j}=1$ to $\mathrm{m}, \mathrm{k}=1$ to $\mathrm{m}$ and $\mathrm{i}=\mathrm{n}$ to 1 ,

Here ejk, $j=1$ to $m$ and $k=1$ to $m$, are the keys in the encryption process, and djk $\mathrm{j}=1$ to $\mathrm{m}$ and $\mathrm{k}=1$ to $\mathrm{m}$, are the corresponding keys in the decryption process. The keys ejk and $\mathrm{djk}$ are related by the relation

$\left(e_{j k} d_{j k}\right) \bmod 256=1$,

that is, $d_{j k}$ is the multiplicative inverse of the given $e_{j k}$. Here it is to be noted that both $e_{j k}$ and $d_{j k}$ are odd numbers which are lying in [1-255].

For convenience, we may write

$\mathrm{E}=\left[\mathrm{e}_{\mathrm{jk}}\right], \quad \mathrm{j}=1$ to $\mathrm{m}$ and $\mathrm{k}=1$ to $\mathrm{m}$.

$\mathrm{D}=\left[\mathrm{d}_{\mathrm{jk}}\right], \quad \mathrm{j}=1$ to $\mathrm{m}$ and $\mathrm{k}=1$ to $\mathrm{m}$. where $\mathrm{E}$ and $\mathrm{D}$ are called as key bunch matrices.

The flow charts describing the encryption and the decryption processes are given by 


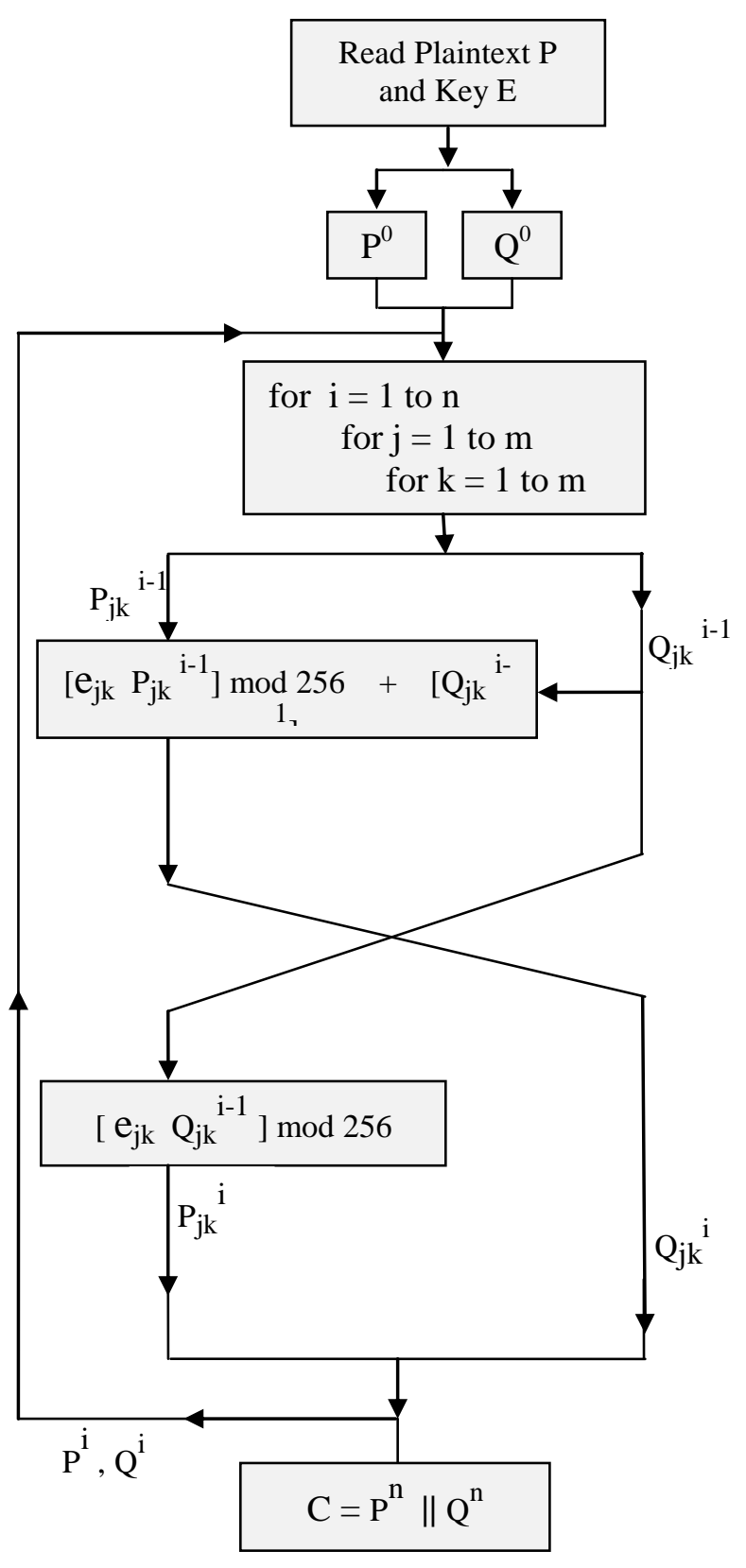

Figure 1. The Process of Encryption

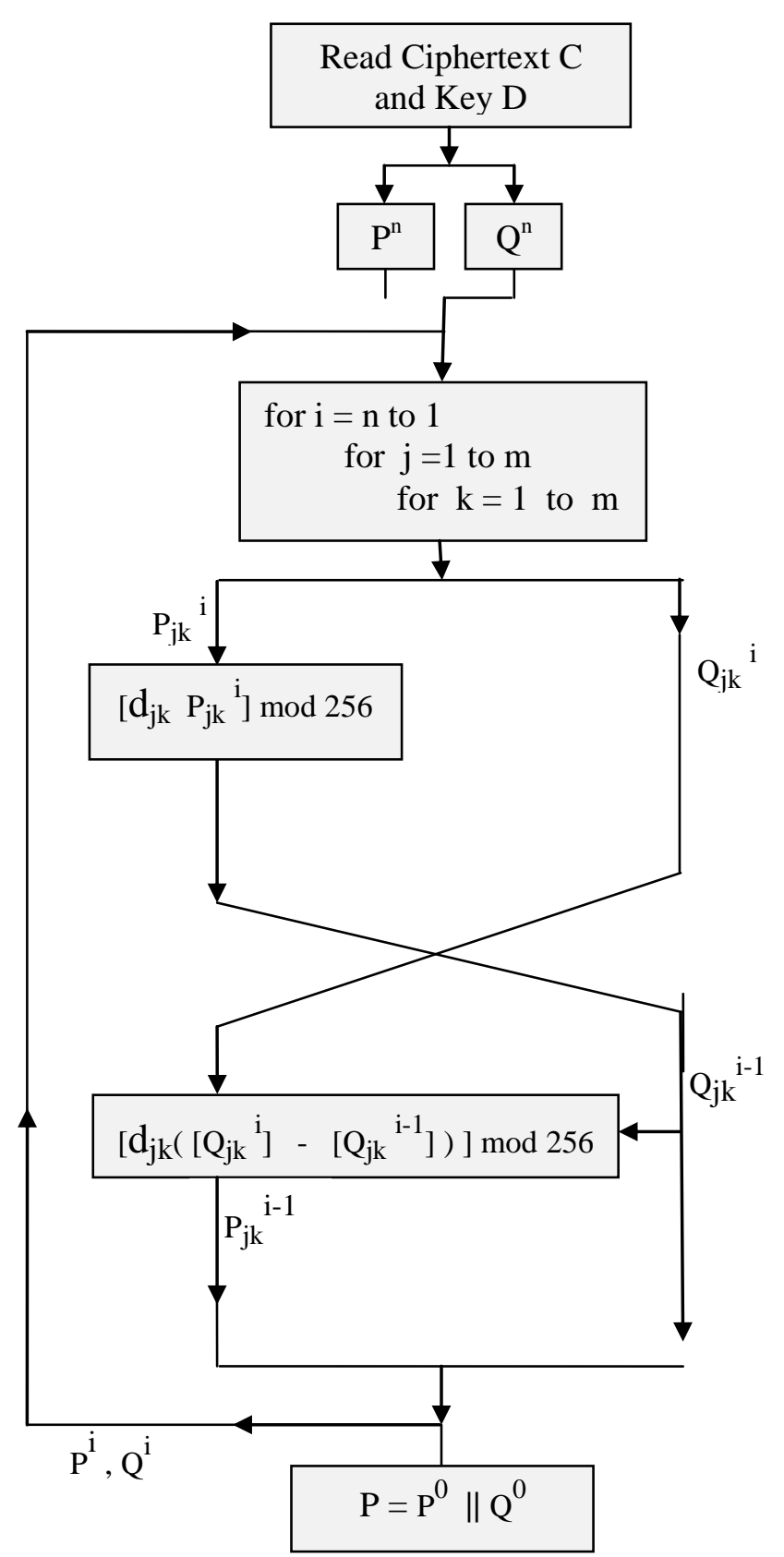

Figure 2. The process of Decryption 
The corresponding algorithms are written in the form given below.

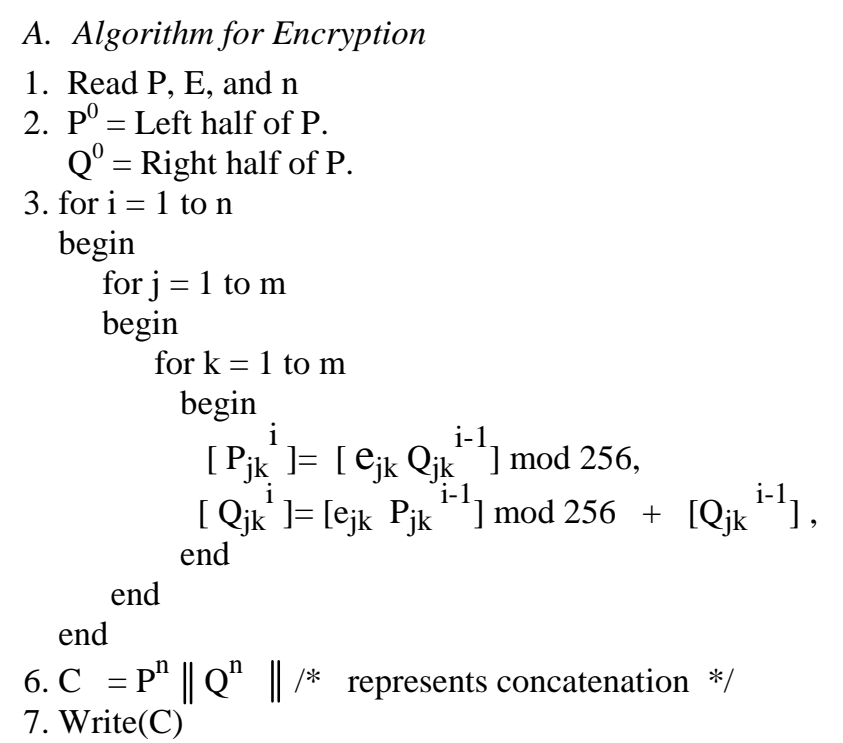

\section{B. Algorithm for Decryption}

1. Read C, D, and $\mathrm{n}$.

2. $P^{n}=$ Left half of $C$

$\mathrm{Q}^{\mathrm{n}}=$ Right half of $\mathrm{C}$

3. for $\mathrm{i}=\mathrm{n}$ to 1

begin

for $\mathrm{j}=1$ to $\mathrm{m}$

begin

for $\mathrm{k}=1$ to $\mathrm{m}$

begin

$\left[\mathrm{Q}_{j k}{ }^{\mathrm{i}-1}\right]=\left[\mathrm{d}_{\mathrm{jk}} \mathrm{P}_{\mathrm{jk}}^{\mathrm{i}}\right] \bmod 256$,
$\left[\mathrm{P}_{\mathrm{jk}}^{\mathrm{i}-1}\right]=\left[\mathrm{d}_{\mathrm{jk}}\left(\left[\mathrm{Q}_{\mathrm{jk}}{ }^{\mathrm{i}}\right]-\left[\mathrm{Q}_{\mathrm{jk}}{ }^{\mathrm{i}-1}\right]\right] \bmod 256\right.$
end

end

end

6. $\mathrm{P}=\mathrm{P}^{0}\left\|\mathrm{Q}^{0} \quad / *\right\|$ represents concatenation */

7. Write $(\mathrm{P})$

\section{ILlUSTRATION OF THE CIPHER}

Consider the plaintext given below

Sister! What a pathetic situation! Father, who joined congress longtime back, he cannot accept our view point. That's how he remains isolated. Eldest brother who have become a communist, having soft corner for poor people, left our house longtime back does not come back to our house! Second brother who joined Telugu Desam party in the time of NTR does not visit us at any time. Our brother in law who is in Bharathiya Janata Party does never come to our house. Mother is very unhappy!

(3.1)

Let us focus our attention on the first 32 characters of the above plaintext. This is given by

Plaintext (3.2)

On using the EBCIDIC code, we obtain

$$
\mathrm{P}=\left[\begin{array}{cccccccc}
083 & 105 & 115 & 116 & 101 & 114 & 033 & 032 \\
087 & 104 & 097 & 116 & 032 & 097 & 032 & 112 \\
097 & 116 & 104 & 101 & 116 & 105 & 099 & 032 \\
115 & 105 & 116 & 117 & 097 & 116 & 105 & 111
\end{array}\right]
$$

This can be written in the form

$$
\mathrm{P}^{0}=\left[\begin{array}{cccc}
083 & 105 & 115 & 116 \\
087 & 104 & 097 & 116 \\
097 & 116 & 104 & 101 \\
115 & 105 & 116 & 117
\end{array}\right]
$$

and

$$
\mathrm{Q}^{0}=\left[\begin{array}{cccc}
101 & 114 & 033 & 032 \\
032 & 097 & 032 & 112 \\
116 & 105 & 099 & 032 \\
097 & 116 & 105 & 111
\end{array}\right]
$$

Let us now take the key bunch matrix $\mathrm{E}$ in the form

$$
E=\left[\begin{array}{cccc}
125 & 133 & 057 & 063 \\
005 & 135 & 075 & 015 \\
027 & 117 & 147 & 047 \\
059 & 107 & 073 & 119
\end{array}\right]
$$

On using the concept of multiplicative inverse, given by the relation (2.5), we get the key bunch matrix D in the form

$$
\mathrm{D}=\left[\begin{array}{cccc}
213 & 077 & 009 & 191 \\
205 & 055 & 099 & 239 \\
019 & 221 & 155 & 207 \\
243 & 067 & 249 & 071
\end{array}\right]
$$

On using (3.4) - (3.6) and applying the encryption algorithm, we get the ciphertext $\mathrm{C}$ in the form

$$
\mathrm{C}=\left[\begin{array}{lllllllll}
036 & 138 & 014 & 142 & 000 & 238 & 090 & 106 \\
110 & 090 & 214 & 104 & 144 & 118 & 246 & 206 \\
016 & 022 & 098 & 018 & 194 & 218 & 070 & 114 \\
108 & 120 & 038 & 118 & 208 & 224 & 146 & 196
\end{array}\right]
$$


On using the ciphertext $\mathrm{C}$ given by (3.8), the key bunch $\mathrm{D}$ given by (3.7), and the decryption algorithm given in section 2 , we get back the original plaintext.

Now let us consider the avalanche effect which predicts the strength of the cipher.

On changing the fourth row, fourth column element of P0 from 117 to 119 , we get a one bit change in the plaintext as the EBCIDIC codes of 117 and 119 are 01110101 and 01110111. On using the modified plaintext and the encryption key bunch matrix $\mathrm{E}$ we apply the encryption algorithm, and obtain the corresponding ciphertext in the form

$$
\mathrm{C}=\left[\begin{array}{cccccccc}
060 & 106 & 182 & 142 & 076 & 198 & 038 & 132 \\
182 & 196 & 242 & 196 & 000 & 034 & 194 & 240 \\
140 & 252 & 088 & 140 & 108 & 090 & 146 & 124 \\
042 & 022 & 094 & 180 & 156 & 250 & 206 & 084
\end{array}\right]
$$

On comparing (3.8) and (3.9) in their binary form, we find that these two ciphertext differ by 129 bits out of 256 bits. This shows the strength of the cipher is quite considerable.

Now let us consider the one bit change in the key, On changing second row, third column element of $\mathrm{E}$ from 75 to 74 , we get a one bit change in the key. On using the modified key, the original plaintext (3.2) and the encryption algorithm, we get the cipher text in the form

$$
\mathrm{C}=\left[\begin{array}{cccccccc}
242 & 248 & 202 & 122 & 058 & 004 & 036 & 154 \\
022 & 252 & 002 & 206 & 104 & 098 & 116 & 002 \\
190 & 108 & 190 & 072 & 250 & 106 & 022 & 200 \\
044 & 114 & 220 & 222 & 050 & 106 & 030 & 220
\end{array}\right]
$$

On comparing (3.8) and (3.10), in their binary form, we find that these two ciphertexts differ by 136 bits out of 256 bits. This also shows that the cipher is expected to be a strong one.

\section{III.CRYPTANALYSIS}

In the literature of the cryptography the strength of the cipher is decided by exploring cryptanalytic attacks. The basic cryptanalytic attacks that are available in the literature [2] are

1) Ciphertext only attack (Brute Force Attack),

2) Known plaintext attack,

3) Chosen plaintext attack, and

4) Chosen ciphertext attack.

In all the investigations generally we make an attempt to prove that a block cipher sustains the first two cryptanalytic attacks. Further, we make an attempt to intuitively find out how far the later two cases are applicable for breaking a cipher.

As the key $\mathrm{E}$ is a square matrix of size $\mathrm{m}$, the size of the key space is
$\left(8 \mathrm{~m}^{2}\right)$
$0.8 \mathrm{~m}^{2}$
$0.8 \mathrm{~m}^{2}$
$2.4 \mathrm{~m}^{2}$

$$
2=\left(2^{10}\right) \quad \approx\left(10^{3}\right) \quad=(10)
$$

If we assume that the time required for the encryption with each key in the key space as 10-7 seconds, then the time required for the execution with all the keys in the key space is

$$
\begin{aligned}
& \left.10^{\left(2.4 \mathrm{~m}^{2}\right)} \times 10^{-7} \times 12 \times 1 \mathrm{~m}^{2}-15\right) \text { years } \\
& 365 \times 24 \times 60 \times 60
\end{aligned}
$$

In the present analysis, as $m=4$, the time required is given by $3.12 \times 1023.4$ years. As this is a formidable quantity we can readily say that this cipher cannot be broken by the brute force approach.

Let us know examine the strength of the known plaintext attack. If we confine our attention to one round of the iteration process, that is if $n=1$, the equations governing the encryption are given by

$\left[\mathrm{P}_{\mathrm{jk}}{ }^{1}\right]=\left[\mathrm{e}_{\mathrm{jk}} \mathrm{Q}_{\mathrm{jk}}^{0}\right] \bmod 256$,

$\left[\mathrm{Q}_{\mathrm{jk}}^{1}\right]=\left[\mathrm{e}_{\mathrm{jk}} \mathrm{P}_{\mathrm{jk}}{ }^{0}\right] \bmod 256+\left[\mathrm{Q}_{\mathrm{jk}}{ }^{0}\right]$,

where, $\mathrm{j}=1$ to $\mathrm{m}$, and $\mathrm{k}=1$ to $\mathrm{m}$.

and

$\mathrm{C}=\mathrm{P}^{1} \| \mathrm{Q}^{1}$

In the case of this attack, as $\mathrm{C}$, yielding $\mathrm{P}_{\mathrm{jk}}{ }^{1}$ and $\mathrm{Q}_{\mathrm{jk}}{ }^{1}$ and as $\mathrm{P}$ yielding $\mathrm{P}_{\mathrm{jk}}{ }^{0}$ and $\mathrm{Q}_{\mathrm{jk}}{ }^{0}$ are known to the attacker, he can readily determine $e_{j k}$ by using the concept of the multiplicative inverse. Thus let us proceed one step further.

On considering the case corresponding to the second round of the iteration $(n=2)$, we get the following equations in the encryption process.

$\left[\mathrm{P}_{\mathrm{jk}}^{1}\right]=\left[\mathrm{e}_{\mathrm{jk}} \mathrm{Q}_{\mathrm{jk}}^{0}\right] \bmod 256$

and

$\left[\mathrm{Q}_{\mathrm{jk}}{ }^{1}\right]=\left[\mathrm{e}_{\mathrm{jk}} \mathrm{P}_{\mathrm{jk}}{ }^{0}\right] \bmod 256+\left[\mathrm{Q}_{\mathrm{jk}}{ }^{0}\right]$,

$\left[\mathrm{P}_{\mathrm{jk}}^{2}\right]=\left[\mathrm{e}_{\mathrm{jk}} \mathrm{Q}_{\mathrm{jk}}{ }^{1}\right] \bmod 256$,

and

$\left[\mathrm{Q}_{\mathrm{jk}}^{2}\right]=\left[\mathrm{e}_{\mathrm{jk}} \mathrm{P}_{\mathrm{jk}}{ }^{1}\right] \bmod 256+\left[\mathrm{Q}_{\mathrm{jk}}{ }^{1}\right]$,

where, $\mathrm{j}=1$ to $\mathrm{m}$ and $\mathrm{k}=1$ to $\mathrm{m}$.

Further we have,

$\mathrm{C}=\mathrm{P}^{2} \| \mathrm{Q}^{2}$.

Here $\mathrm{P}_{\mathrm{jk}}{ }^{0}$ and $\mathrm{Q}_{\mathrm{jk}}{ }^{0}$ are known to us, as $\mathrm{C}$ is known. We also know $\mathrm{P}_{\mathrm{jk}}{ }^{0}$ and $\mathrm{Q}_{\mathrm{jk}}{ }^{0}$ as this is the known plaintext attack. But here, we cannot know $\mathrm{P}_{\mathrm{jk}}{ }^{1}$ and $\mathrm{Q}_{\mathrm{jk}}{ }^{1}$ either from the forward side or from the backward side. Thus $\mathrm{e}_{\mathrm{jk}}$ cannot be determined by 
any means, and hence this cipher cannot be broken by the known plaintext attack.

As the equations governing the encryption are complex, it is not possible to intuitively either a plaintext or a ciphertext and attack the cipher. Thus the cipher cannot be broken by the last two cases too. Hence we conclude that this cipher is a very strong one.

\section{COMPUTATIONS AND CONCLUSIONS}

In this investigation we have developed a block cipher by modifying the Feistel cipher. In this analysis the modular arithmetic addition plays a fundamental role. The key bunch encryption matrix $\mathrm{E}$ and the key bunch decryption matrix D play a vital role in the development of the cipher. The computations involved in this analysis are carried out by writing programs in $\mathrm{C}$ language.

On taking the entire plaintext (3.1) into consideration, we have divided it into 14 number of blocks. In the last block, we have included 26 blanks characters to make it a complete block. On taking the encryption key bunch $\mathrm{E}$ and carrying out the encryption of the entire plaintext, by applying encryption algorithm given in section 2 , we get the ciphertext $\mathrm{C}$ in the form given below

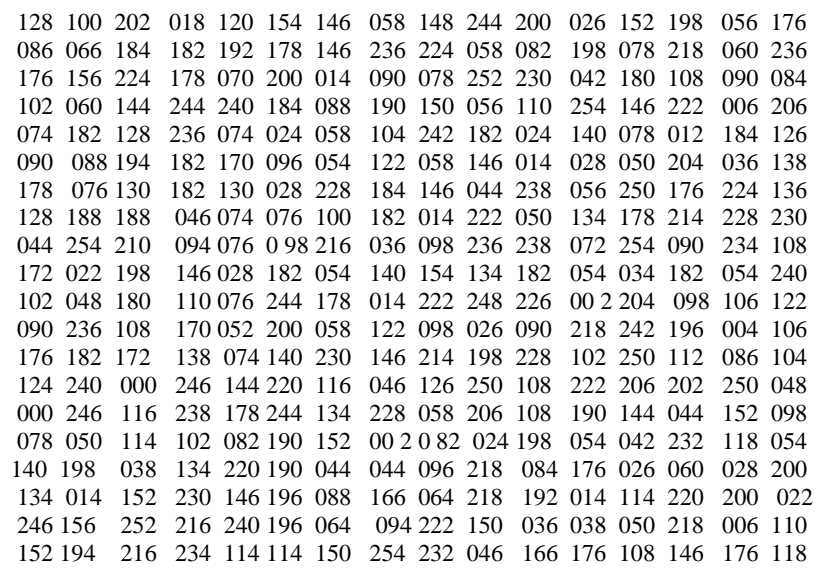

$\begin{array}{llllllllllllllll}246 & 036 & 254 & 044 & 244 & 054 & 214 & 138 & 098 & 072 & 142 & 090 & 154 & 198 & 076 & 066\end{array}$ $\begin{array}{llllllllllllllll}218 & 154 & 144 & 090 & 026 & 248 & 178 & 024 & 218 & 182 & 038 & 250 & 088 & 006 & 110 & 124\end{array}$ $\begin{array}{llllllllllllllll}240 & 000 & 102 & 048 & 180 & 188 & 172 & 118 & 054 & 212 & 176 & 104 & 080 & 156 & 242 & 070\end{array}$ $\begin{array}{llllllllllllllll}214 & 198 & 228 & 102 & 250 & 092 & 228 & 190 & 250 & 074 & 020 & 102 & 152 & 006 & 110 & 076\end{array}$

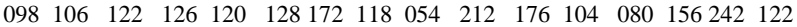

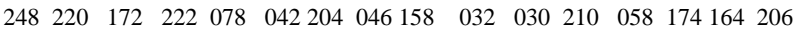

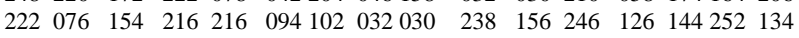

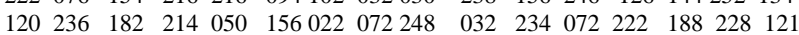

In this we have excluded the ciphertext which is already presented in (3.8)

In the light of this analysis, here we conclude that this cipher is an interesting one and a strong one, and this can be used for the transmission of any information through internet.

\section{REFERENCES}

[1] V.U.K Sastry and K. Anup Kumar " A Novel Feistel Cipher Involving a bunch of Keys Supplemented with XOR Operation" (IJACSA) International Journal of Advanced Computer Science and Applications, 2012.

[2] William Stallings, Cryptography and Network Security, Principles and Practice, Third Edition, Pearson, 2003.

\section{AUthors PROFILE}

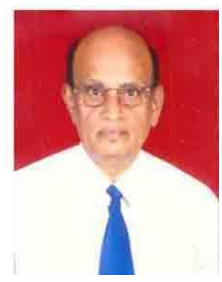

Dr. V. U. K. Sastry is presently working as Professor in the Dept. of Computer Science and Engineering (CSE), Director (SCSI), Dean (R \& D), SreeNidhi Institute of Science and Technology (SNIST), Hyderabad, India. He was Formerly Professor in IIT, Kharagpur, India andWorked in IIT, Kharagpurduring 1963 - 1998. He guided 12 $\mathrm{PhDs}$, and published more than 40 research papers in various international journals. His research interests are Data Mining and Genetic Algorithms. Network Security \& Cryptography, Image Processing,

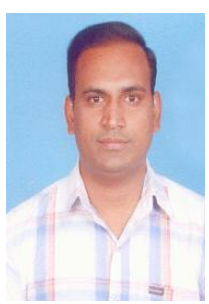

Mr. K. Anup Kumar is presently working as an Associate Professor in the Department of Computer Science and Engineering, SNIST, Hyderabad India. He obtained his B.Tech (CSE) degree from JNTU Hyderabad and his M.Tech (CSE) from Osmania university, Hyderabad. He is now pursuing his $\mathrm{PhD}$ from JNTU, Hyderabad, India, under the supervision of Dr. V.U.K. Sastry in the area of Information Security and Cryptography. He has 10 years of teaching experience and his interest in research area includes, Cryptography, Steganography and Parallel Processing Systems. 